... Peter Meier-Abt: Internist, Klinischer Pharmakologe und Präsident der Schweizerischen Akademie der Medizinischen Wissenschaften (SAMW)

\title{
«Mein Herz schlägt für die Forschung und die Klinik»
}

Daniel Lüthi

Text und Bilder

danielluethi[at]gmx.ch
Es gäbe mehrere Büros, wo wir uns unterhalten könnten, mehrere Orte auch für ein typisches Foto. Für unser Gespräch käme beispielsweise das Wildt'sche Haus in Frage, die ehemalige Villa einer reichen Färberfamilie, ein aristokratisch anmutendes, repräsentatives Haus an prominenter Lage. Es ist der Sitz der Schweizerischen Akademie der Medizinischen Wissenschaften (SAMW), die Peter Meier-Abt seit einem Jahr präsidiert.

\section{Verschiedene Welten}

Der Ort trägt seinen Vornamen: Petersplatz. «Von wegen Aristokrat», sagt er jetzt, wo wir vornedran stehen, «eher bin ich ein Bauer. Beide Grosseltern hatten einen Landwirtschaftsbetrieb, dort fühlte ich mich zu Hause.» Wir gehen weiter und setzen uns an den Tisch eines etwas muffigen Zimmers in einem renovationsbedürftigen Altstadthaus aus dem 13. Jahrhundert. Hier, am Heuberg, ist Meier-Abt seit seinem

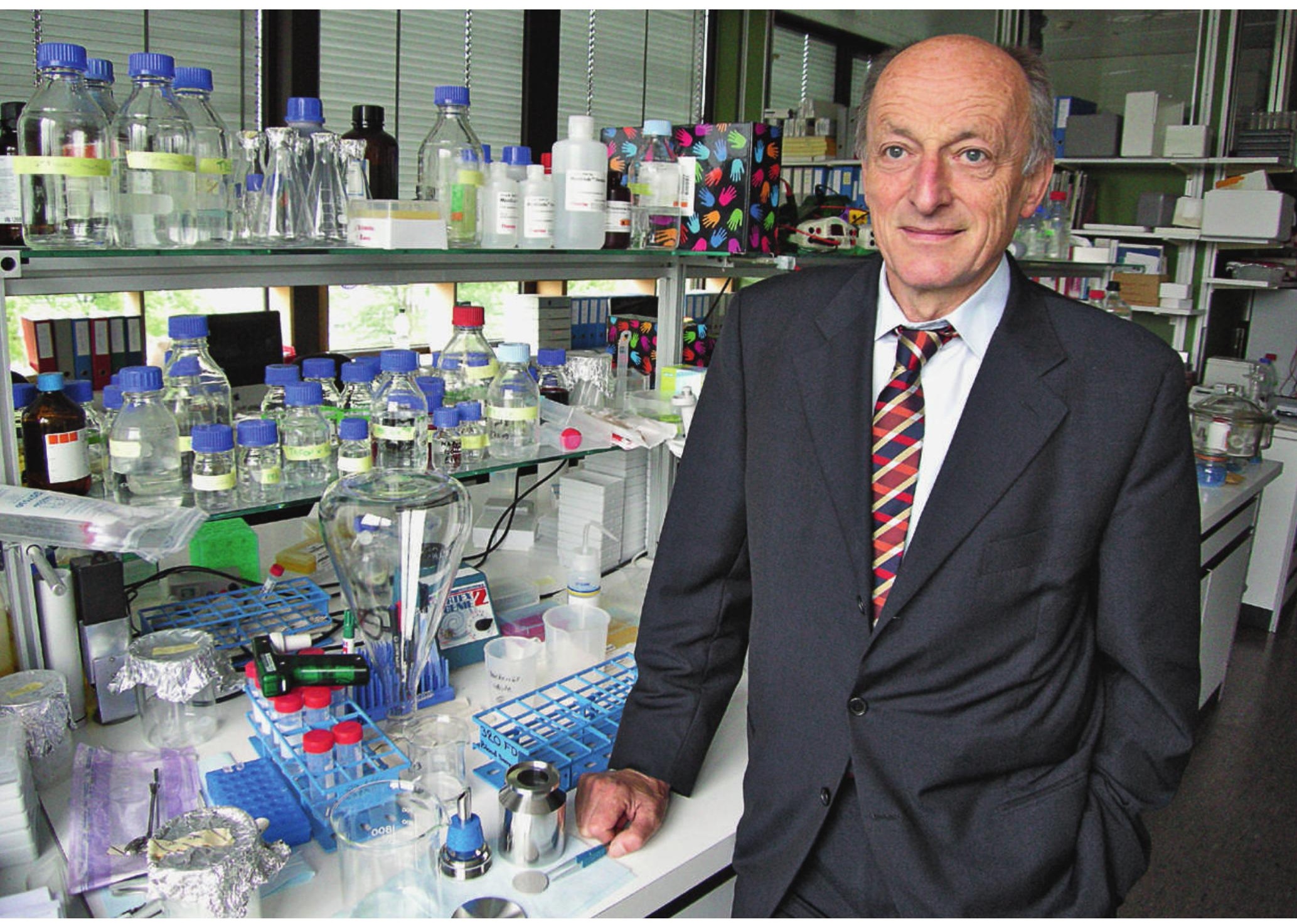


Rücktritt als Vizerektor noch für die Universität Basel tätig. Für das Foto wählen wir schliesslich das «Zentrum für Lehre und Forschung» am Universitätsspital, hier ist das Departement für Biomedizin beheimatet, das er ad interim leitet. Das Bild machen wir aber weder im hiesigen Büro noch in einem der Hörsäle, die durchaus auch repräsentativ gewesen wären, sondern in einem Labor. Es ist ein Gang back to the roots. «Hier fühle ich mich wohl», sagt der distinguierte Herr, der mit seiner Kleidung gar nicht so recht in diese Umgebung passen will. Der Gegensatz ist also nur ein scheinbarer, «in solchen Räumen habe ich mein halbes Leben verbracht.»

Unser Gang durch die verschiedenen Wirkungsorte ist symptomatisch: für die professionelle Vielseitigkeit von Meier-Abt, aber auch für seinen gegenwärtigen Lebensabschnitt. Er befindet sich in einer Übergangszeit, «in einer Transitionsphase», wie er sich als Wissenschaftler ausdrückt. Eben erst ist er 65 geworden: «Ich bin daran, das professionelle Leben loszulassen. Den wichtigsten und schwierigsten Schritt in diesem Prozess machte ich allerdings bereits 2005. Damals verliess ich meine Forschungsgruppe in Zürich.»

\section{Mediziner und Biochemiker}

Die Forschung also liegt ihm besonders am Herzen. Zentrales Organ dabei war immer die Leber. «Seit meinen Studienjahren interessiert mich die Frage, wie Medikamente in die Leber transportiert, abgebaut und dann wieder ausgeschieden werden. Und ganz besonders die Pharmakogenetik, also die genetisch bedingten Unterschiede von Arzneimittelwirkungen bei verschiedenen Menschen.» Es folgt ein kurzer wissenschaftlicher Vortrag, der für den NichtSpezialisten ziemlich unverständlich ist. Stichworte daraus: Gallebildung, Membrantransport, oxidativer Arzneimittelmetabolismus, Lipide, polarisierte Moleküle, Gallensäuren, sequenzierte Transportproteine. «Immer schon war ich nebst Mediziner auch Biochemiker», erläutert Meier-Abt. Und wird dann plötzlich ganz verständlich: «Jedes Medikament ist ein Fremdstoff im menschlichen Körper und muss in der Leber «entgiftet werden. Deshalb sagte schon Paracelsus: ¿Die Dosis entscheidet, ob ein Medikament ein Heilmittel oder ein Gift ist.»»

Vernetzungen und Brückenbildungen sind wichtig, sagt er: «Wie können wir die Grundlagenforschung besser mit der klinischen Forschung und diese wiederum mit der Praxis, also der Versorgungsforschung, verbinden - und umgekehrt? Dieser interdisziplinäre Ansatz, diese Interaktionen, diese Aspekte einer translationalen Forschung interessieren mich.» Konkretes Beispiel: «Ausgehend von klinischen Beobachtungen haben wir in Tierversuchen erforscht, wie Grapefruitsaft oder Johanniskrautextrakt die Aufnahme von gewissen Arzneimitteln, zum Beispiel von Zyklosporin, in den Körper hemmen.»

Ein mögliches Resultat solch vernetzter Forschung ist die personalisierte Medizin, «das sind auf

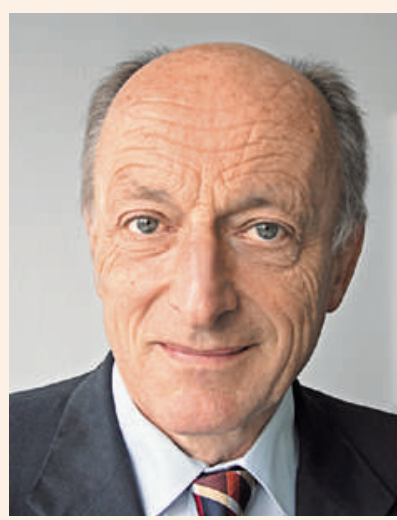

\section{Peter Meier-Abt}

Prof. Dr. med. Peter Meier-Abt wurde 1947 in Baden geboren und wuchs in Lengnau (AG) auf. Er studierte Medizin an den Universitäten Freiburg und Basel und schloss 1974 mit dem Staatsexamen ab. Seine Weiterbildung zum Facharzt für Innere Medizin sowie für Klinische Pharmakologie und Toxikologie absolvierte er in Basel, Zürich und in den USA. 1984 übernahm er die Leitung der Abteilung für Klinische Pharmakologie und Toxikologie am Universitätsspital Zürich; 1992 wurde er zum ordentlichen Professor an der Universität Zürich ernannt.

Von 2005 bis letzten Juli war Prof. Meier-Abt Vizerektor der Universität Basel. Er betreute die Bereiche Forschung und Nachwuchsförderung inkl. Doktoratsausbildung, EU-Research, sowie Wissens- und Technologie-Transfer. Seit einem Jahr ist er Präsident der Schweizerischen Akademie der Medizinischen Wissenschaften (SAMW). 2004-2011 gehörte er dem SAMW-Vorstand als Vizepräsident an. Peter Meier-Abt ist verheiratet und Vater von zwei erwachsenen Töchtern. Er lebt in Basel und Zürich - und bald schon in Beckenried am Vierwaldstättersee.

das Individuum abgestimmte, massgeschneiderte therapeutische Massnahmen.» Erklärung dazu: «Jeder Mensch ist genetisch anders als der andere. Deshalb ist es auch so, dass verschiedene Personen auf Medikamente verschieden reagieren und sie unterschiedlich schnell abbauen. Das wiederum heisst aber: Das Spektrum einer Arzneimitteltherapie reicht von einer möglichen Vergiftung bis zur völligen Wirkungslosigkeit. Ziel ist es deshalb, dass wir mittels molekularer Biomarker möglichst genau jene Personen identifizieren können, die optimal auf eine spezifische therapeutische Massnahme ansprechen. In Zukunft wird es darum gehen, bei bestimmten Krankheiten möglichst viele genetische und epigenetische Daten - sogenannte Genotypen - zu sammeln und mit den klinischen Symptomen, den Phänotypen, zu korrelieren. Aus solchen Daten kann man dann diagnostische «Chips» entwickeln, die bei 
spezifischen Krankheiten eine individualisierte Therapie erlauben.»

Spricht so ein Arzt? «Ja, sicher auch», schmunzelt der Professor, schliesst die Augen, überlegt. «Ein halbes Jahr lang habe ich seinerzeit als stellvertretender Landarzt im Glarnerland gearbeitet. Das gefiel mir, aber meine Familie wollte in die Stadt. Ich musste mich also entscheiden. So wurde ich zum physician-scientist und das bin ich noch heute. Mein Herz schlägt für die Forschung und die Klinik.»

\section{Evidenz als Grundlage}

Physician-scientist: Der Begriff fällt immer wieder. Und er hängt eng mit einem anderen Credo von Peter Meier-Abt zusammen, nämlich dem Prinzip der Evidenz-basierten Medizin. «Ärztliche Massnahmen müssen auf wissenschaftlichen Erkenntnissen abgestützt sein, ihre Wirksamkeit muss nachgewiesen sein. Und: Ab und zu müssen wir auch ehrlich genug sein und akzeptieren, dass es Dinge gibt, die wir nicht wissen. Wissenschaftliche Evidenz vorzutäuschen, wo es sie nicht gibt, widerspricht den Prinzipien der medizinischen Ethik», sagt Meier-Abt mit Blick auf die Tätigkeit, die ihn von jetzt an schwergewichtig beschäftigen wird, nämlich das Präsidium der SAMW. «Es ist beeindruckend, was die SAMW im Bereich der Ethik alles schon geleistet hat. Wichtig dabei ist unter anderem, dass wir bei unseren ethischen Erwägungen nie aus falschen Beweggründen eine anti-wissenschaftliche Haltung einnehmen.»

\section{«Es kann nicht sein, dass die Kluft zwischen Natur- wissenschaften und Klinik immer grösser wird.»}

Eines der Hauptthemen in seiner Funktion als SAMW-Präsident ist für Meier-Abt die Nachwuchsförderung. Zum Beispiel, wen wundert's: «Die jungen Mediziner sollen für die Forschung motiviert werden. Sie sollen lernen, mit genetischen und epigenetischen Daten und modernen IT-Technologien umzugehen. Es kann nicht sein, dass die Kluft zwischen Naturwissenschaften und Klinik immer grösser wird, dass sich ein Biologe und ein Arzt letztlich nicht mehr verstehen.»
Und wie steht es mit der Hausarztmedizin? Unmissverständlich stellt Meier-Abt fest: «Ja, wir müssen die Hausarztmedizin fördern. Bloss: Sollen wir dafür einfach mehr Leute ausbilden? Oder müssten wir nicht vor allem neue Versorgungsmodelle definieren? Junge Ärztinnen und Ärzte wollen im Team arbeiten. Auch deshalb sind hier wahrscheinlich regionale, integrative Versorgungsnetze die Zukunft, zu denen nicht nur Ärzte, sondern beispielsweise auch Pflegende, Physiotherapeuten oder Pharmazeuten gehören.» Dazu komme ein gesellschaftliches Element: «Die Ausbildung eines Mediziners oder einer Medizinerin kostet den Steuerzahler rund eine halbe Million Franken - bei voller Freiheit für die ausgebildete Person. Ich denke, wir müssten die Ausbildung mit einer Verpflichtung zum Praktizieren verbinden. Und wir müssten zuerst einmal eine Gesamtstrategie der künftigen Versorgung erarbeiten dann könnten wir auch den jeweiligen Bedarf in den verschiedenen Disziplinen definieren.»

Zum Arzt, Forscher, Forschungsmanager und Wissenschaftler kommt jetzt also noch der Politiker Meier-Abt.

\section{Wachstum und Limiten}

Wichtiger werden jetzt, im Zeitalter der Pension, des aktiven Unruhestandes, auch die eigenen, die ganz persönlichen Bedürfnisse und Gedanken. So befasst sich Peter Meier-Abt nicht nur theoretisch und als Funktionär mit Fragen rund um Leben und Tod. «Mit meiner eigenen Vergänglichkeit habe ich mich abgefunden», sagt er. «Aber so lange ich noch aktiv sein kann, will ich aktiv sein. Drei Mal pro Woche laufen, schwimmen oder velofahren, das brauche ich.»

Mehr als das eigene Ende beschäftige ihn ein mögliches Ende dieser Welt. «Diese grenzenlose, rücksichtslose Wachstumseuphorie macht mir Sorgen. Gehen Sie mal in einen Schlachthof - und dann ins Shoppingcenter - und schauen Sie, wie viel Fleisch weggeworfen wird.»

Wenn schon Wachstum, dann bezüglich Erkenntnis, sinniert der alternde, aber junggebliebene Professor. «Die Biologie hilft uns, das Leben besser zu verstehen. Und sie hilft uns auch, unsere Limiten zu erkennen. Aber die Biologie ist komplex - je genauer wir hinsehen, umso grösser werden die Unschärfen.»

Am Ende jeden Monats stellt die Schweizerische Ärztezeitung eine Persönlichkeit vor, die sich im Gesundheitswesen engagiert. Im Juli schildert Daniel Lüthi seine Begegnung mit Roberto Malacrida, Professor für Intensivmedizin, Klinische Ethik und Medical Humanities. 